\title{
Genome gazing in ammonia-oxidizing archaea
}

\author{
Emiley A. Eloe-Fadrosh
}

This month's Genome Watch highlights the unique evolutionary history, metabolic functions, and newly identified viruses and associated mobile genetic elements for the highly abundant and ubiquitous ammonia-oxidizing archaea.

The discovery of ammonia-oxidizing archaea (AOA) nearly 15 years ago has greatly advanced our understanding of global nitrogen and carbon cycling, as well as the ecology and evolution of the domain Archaea. Rampant molecular surveys of the canonical functional marker gene, amoA, which encodes the subunit A of the key metabolic enzyme ammonia monooxygenase, have uncovered the sheer abundance and ubiquity of these important microorganisms and have yielded insights into patterns of ecosystem partitioning, niche specialization and hypothesized ecophysiological adaptations ${ }^{1}$. More recently, genomic insights from isolated representatives, enrichment cultures and an expanding collection of single-cell genomes and metagenome-assembled genomes have enabled a broader view of the unique evolutionary history of AOA.

Ren et al. $^{2}$ leveraged a genome-based molecular dating analysis with more than 60 AOA genomes to associate thaumarchaeal evolution with geological timescales. Through detailed phylogenomic reconstructions, diversification of terrestrial AOA from non-AOA Thaumarchaea was found

to coincide with the Great Oxygenation Event estimated at 2,300 million years ago. Metabolic consequences of this

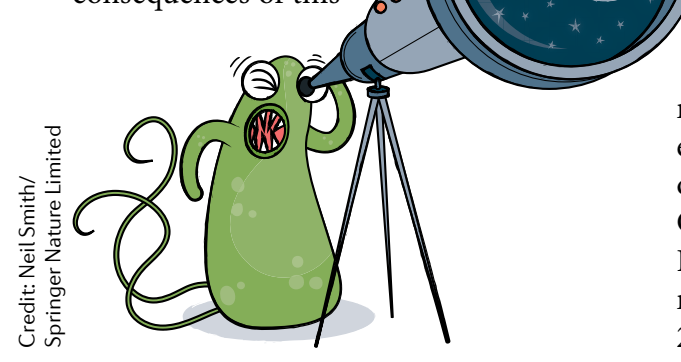

transition included gain of aerobic energy production via ammonia oxidation and cobalamin and biotin biosynthetic capacity, with complementary loss of anaerobic carbon fixation through the Wood-Ljungdahl pathway and dissimilatory nitrate and sulfate reduction. Similarly, the phylogenomic dating suggested that habitat expansion of terrestrial AOA to marine shallow and deep sites was likely to be associated with acquiring genes via horizontal transfer to regulate osmotic pressure (for example, the operon ect $A B C D$ which encodes genes involved in the biosynthesis of the compatible solute ectoine).

Beyond tracking the evolutionary history of AOA, Kitzinger et al. ${ }^{3}$ recently demonstrated direct and indirect utilization of urea and cyanate by marine Thaumarchaea through field measurements and experimental validation with the model isolate Nitrosopumilus maritimus. Interestingly, metagenomic analysis revealed that only a subpopulation of Thaumarchaea in marine waters encoded ureases and that there was no evidence of encoded cyanases. Thus, although genomic data can provide a fruitful avenue to predict functional and metabolic properties of AOA, there is still much to be learned about the ecosystem functioning and niche specialization.

Our expanding view of AOA functional and metabolic diversity is similarly mirrored with a broader appreciation for integrated mobile genetic elements (iMGEs), including viruses, conjugative elements, small cryptic plasmids, transposable elements and casposons, which are the recently described transposon-like elements with a CRISPR-associated Cas1 protein homologue. Krupovic et al. ${ }^{4}$ analysed 21 genomes of thaumarchaeal species and unveiled that nearly $2 \mathrm{Mbp}$ of mobile DNA consists of an expansive suite of iMGE-specific genes, stress response genes presumably important for host adaptation to new environments and auxiliary metabolic genes (AMGs). The observation of diverse AMGs, particularly multicopper oxidases, $a m o C$ genes and nitrogen regulatory components, suggests potential modulation of nitrogen metabolism. Indeed, the identification of ecologically distinct virus populations encoding $a m o C^{5}$ highlights the potential global impact of these viruses on nitrogen cycling.

In summary, these studies underscore the power of genome-based inquiry to expand our understanding of archaeal evolutionary history. In addition, such studies can help form hypotheses that can be further tested using experimental model systems. Importantly, the exploration of virus-host interactions and further elucidation of the impact of iMGEs will undoubtedly lead to novel insights into the unique evolutionary trajectories of these biogeochemically important archaea.

$$
\begin{array}{r}
\text { Emiley A. Eloe-Fadrosh } \\
\text { DOE Joint Genome Institute, Walnut Creek, CA, USA. } \\
\text { e-mail: JGI-Microbe@lbl.gov } \\
\text { https://doi.org/10.1038/s41579-019-0248-8 }
\end{array}
$$

1. Alves, R. J. E. et al. Unifying the global phylogeny and environmental distribution of ammonia-oxidizing archaea based on amoA genes. Nat. Commun. 9 1517 (2018).

2. Ren, M. et al. Phylogenomics suggests oxygen availability as a driving force in Thaumarchaeota evolution. ISME J. https://doi.org/10.1038/ s41396-019-0418-8 (2019).

3. Kitzinger, K. et al. Cyanate and urea are substrates for nitrification by Thaumarchaeota in the marine environment. Nat. Microbiol. 4, 234-243 (2019).

4. Krupovic, M. et al. Integrated mobile genetic elements in Thaumarchaeota. Environ. Microbiol. 6, 2056-2078 (2019).

5. Ahlgren, N. A. et al. Discovery of several novel, widespread, and ecologically distinct marine Thaumarchaeota viruses that encode $\mathrm{amoC}$ nitrification genes. ISME J. 13, 618-631 (2019).

Competing interests

The author declares no competing interests. 